\title{
Flare Stars in a Field Near the North Galactic Pole: ROSAT and Optical Observations
}

\author{
G.A. Richter ${ }^{1}$, H.-J. Bräuer ${ }^{1}$, J. Greiner ${ }^{2}$ \\ 1 Sternwarte Sonneberg, 96515 Sonneberg, Germany \\ 2 Max-Planck-Institut für Extraterrestrische Physik, 85740 Garching, Germany
}

In a field of 100 square degrees around $26 \mathrm{Com}, 238 \mathrm{X}$-ray sources have been discovered by ROSAT during the All-Sky-Survey observations. These sources were identified using objective prism spectrograms taken with the Hamburg Schmidt telescope on Calar Alto (courtesy N. Bade) and including the positional accuracy of typically $30^{\prime \prime}$ and the $\mathrm{X}$-ray to optical luminosity ratio. In an ongoing investigation of these X-ray sources on more than 400 plates of the Sonneberg astrographs $400 / 1600 \mathrm{~mm}$ and $400 / 2000 \mathrm{~mm}$, a total of 5 of the M type counterparts were found to be flare stars. In Table 1 we give the ROSAT name (column 1), the new designation as a Sonneberg variable (2), the position of the optical counterpart (3), the magnitude range of the newly discovered flare stars (4), the ROSAT PSPC countrate during the All-Sky-Survey (5), the distance D between X-ray and optical position (6). All 5 objects had not been known to be flaring. The real amplitudes must be larger than those given in column 3 because the (unknown) duration of the flares is usually much shorter than the exposure time of the plates. The figures give APM finding charts of these new flare stars.

The following are remarks on the brightness behaviour of the individual objects (in parentheses: exposure time of the plate in min.; estimated magnitudes):

RX J1235.5+1954: Eclipsing star with flaring activity. Preferred magnitude level about $15^{\mathrm{m}}$. Flares: $2438817.660\left(60 ; 14^{\mathrm{m}} 6\right), 2442513.449\left(140 ; 14^{\mathrm{m}} 6\right)$, $6121.582\left(30 ; 14^{\mathrm{m}} 5\right), 6881.447\left(20 ; 14^{\mathrm{m}} 6\right)$. Minima near $15^{\mathrm{m}} 5$ : 2438106.464 , 8142.581, $2445077.426,7262.415,7566.592$.

RX J1253.6+2247: Eclipsing star with flaring activity. Preferred magnitude about $16^{\mathrm{m}}$. Flares: $2437822.417\left(40 ; 15^{\mathrm{m}} 7\right), 2445056.400\left(30 ; 15^{\mathrm{m}} 5\right)$, $8683.507\left(25 ; 15^{\mathrm{m}}\right)$. Observed minima near 16.5 : $2438083.523, .566,9616.380$, $2444367.370, .384,4636.657,7566.592,7672.411$.

RX J1256.8+2329: Eastern component of two stars of similar magnitude and colour. Observed flares: $2437668.641\left(60 ; 13^{\mathrm{m}} 5\right), 8088.536\left(60 ; 14^{\mathrm{m}} 5\right)$, $9205.483\left(40 ; 14^{\mathrm{m}}\right), 9592.342\left(60 ; 14^{\mathrm{m}}\right), 9945.500\left(40 ; 14^{\mathrm{m}}\right), 2440981.514(40 ;$ $\left.14^{\mathrm{m}}\right), 2107.533\left(20 ; 15^{\mathrm{m}}\right), 4663.524\left(40 ; 14^{\mathrm{m}} 5\right), 6552.376\left(20 ; 15^{\mathrm{m}}\right)$.

RX J1258.9+2112: Eclipsing star with flares. Preferred magnitude level about $17^{\mathrm{m}}$. Flares: $2437790.418\left(60 ; 15^{\mathrm{m}} 5\right), 8851.470\left(60 ; 16^{\mathrm{m}}\right), 9537.451(60$; $\left.16^{\mathrm{m}}\right), 2445815.404\left(18 ; 16^{\mathrm{m}}\right), 5820.389(18 ; 15 \mathrm{~m} 5)$. Observed minima (about $\left.17^{\mathrm{m}} 5\right): 2437765.468,8084.545,9535.592,9618.504$. 
RX J1300.5+2255: Numerous flares: $2437764.405\left(60 ; 16^{\mathrm{m}} 5\right), 7783.440$ $\left(24 ; 15^{\mathrm{m}}\right), 7790.459\left(60 ; 15^{\mathrm{m}} 5\right), 7822.445\left(40 ; 16^{\mathrm{m}} 5\right), 7823.455\left(60 ; 16^{\mathrm{m}} 5\right), 9611.507$ $\left(60 ; 16^{\mathrm{m}} 5\right), 2442513.449\left(100 ; 16^{\mathrm{m}} 5\right), 4342.372\left(20 ; 16^{\mathrm{m}} 5\right), 5384.628\left(60 ; 16^{\mathrm{m}}\right)$, $6534.403\left(18 ; 15^{\mathrm{m}} 5\right), 7613.457\left(14 ; 16^{\mathrm{m}}\right), 8357.474\left(22 ; 16^{\mathrm{m}}\right)$.

Table 1. Newly discovered flare stars in the Coma field

\begin{tabular}{cccccr}
\hline ROSAT Name & $\begin{array}{c}\text { Variable } \\
\text { Star } \\
\text { designation }\end{array}$ & $\begin{array}{c}\text { Position of } \\
\text { flare star } \\
(2000.0)\end{array}$ & $\begin{array}{c}\text { Magnitude } \\
\text { range (pg) }\end{array}$ & $\begin{array}{c}\text { X-ray } \\
\text { intensity } \\
\text { (cts/sec) }\end{array}$ & D \\
\hline RX J1235.5+1954 & S10935 & $12^{\mathrm{h}} 35^{\mathrm{m}} 32^{\mathrm{s}} .9+19^{\circ} 54^{\prime} 43^{\prime \prime}$ & $14.5-15.5$ & 0.018 & $17^{\prime \prime}$ \\
RX J1253.6+2247 & $\mathrm{S} 10936$ & $12^{\mathrm{h}} 53^{\mathrm{m}} 36^{\mathrm{s}} .2+22^{\circ} 47^{\prime} 35^{\prime \prime}$ & $15.0-16.5$ & 0.075 & $2^{\prime \prime}$ \\
RX J1256.8+2329 & $\mathrm{S} 10937$ & $12^{\mathrm{h}} 56^{\mathrm{m}} 52^{s} .2+23^{\circ} 29^{\prime} 50^{\prime \prime}$ & $13.0-15.5$ & 0.071 & $5^{\prime \prime}$ \\
RX J1258.9+2112 & $\mathrm{S} 10938$ & $12^{\mathrm{h}} 59^{\mathrm{m}} 00^{\mathrm{s}} .3+21^{\circ} 12^{\prime} 56^{\prime \prime}$ & $15.5-17.5$ & $\mathbf{0 . 0 2 9}$ & $15^{\prime \prime}$ \\
RX J1300.5+2255 & $\mathrm{S} 10939$ & $13^{\mathrm{h}} 00^{\mathrm{m}} 33^{\mathrm{s}} .2+22^{\circ} 55^{\prime} 41^{\prime \prime}$ & $15.0-17.0$ & 0.010 & $27^{\prime \prime}$ \\
\hline
\end{tabular}

At the same time there are another 6 known flare stars in the field covered by our investigation. For two of these, finding charts are available, and a check of the coordinates given in the GCVS revealed these to be wrong by $\sim 8^{\prime}$ (CT Com, Haro 1968) and $\sim 10^{\prime}$ (CV Com, Argue \& Kenworthy 1968), respectively. Using the new positions, both sources are not detected during the ROSAT All-SkySurvey. The other four sources (GCVS positions) have also not been detected in $\mathrm{X}$-rays by ROSAT. However, since no finding charts are available for these stars, we have to assume that the coordinates given in the discovery papers (references see GCVS) are correct. The upper limit for the X-ray intensity of all the sources is $0.01 \mathrm{cts} / \mathrm{s}$ in the $0.1-2.4 \mathrm{keV}$ range. These flare stars are listed in Table 2. The magnitude range and the coordinates for the four stars without available finding charts were taken from the GCVS, the latter have been precessed to equinox 2000 .

Table 2. Flare stars not detected in the ROSAT Survey

\begin{tabular}{|c|c|c|c|c|}
\hline \multirow[t]{2}{*}{ Star } & \multicolumn{2}{|c|}{ coordinates $(2000.0)$} & \multirow{2}{*}{$\begin{array}{l}\text { Magnitude } \\
\text { range }(\mathrm{pg})\end{array}$} & \multirow{2}{*}{$\begin{array}{l}\text { Comment on } \\
\text { coordinate }\end{array}$} \\
\hline & R.A. & DEC. & & \\
\hline HO Com & $12^{\mathrm{h}} 19^{\mathrm{m}} 32^{\mathrm{s}}$ & $+25^{\circ} 13^{\prime} 3$ & $16.2-21.0$ & from GCVS \\
\hline CT Com & $12^{\mathrm{h}} 23^{\mathrm{m}} 49^{\mathrm{s}} .0$ & $+24^{\circ} 07^{\prime} 56^{\prime \prime}$ & $12.5-16.0$ & newly determined \\
\hline HQ Com & $12^{\mathrm{h}} 25^{\mathrm{m}} 12^{\mathrm{s}}$ & $+23^{\circ} 36^{\prime} 4$ & $14.7-19.6$ & from GCVS \\
\hline CV Com & $12^{\mathrm{h}} 24^{\mathrm{m}} 26^{\mathrm{s}} .6$ & $+25^{\circ} 45^{\prime} 07^{\prime \prime}$ & $14.3-16.8$ & newly determined \\
\hline HS Com & $12^{\mathrm{h}} 26^{\mathrm{m}} 24^{\mathrm{s}}$ & $+25^{\circ} 54^{\prime} .5$ & $15.1-21.0$ & from GCVS \\
\hline HV Com & $12^{\mathrm{h}} 31^{\mathrm{m}} 53^{\mathrm{B}}$ & $+25^{\circ} 09^{\prime} 4$ & $15.3-16.6$ & from GCVS \\
\hline
\end{tabular}



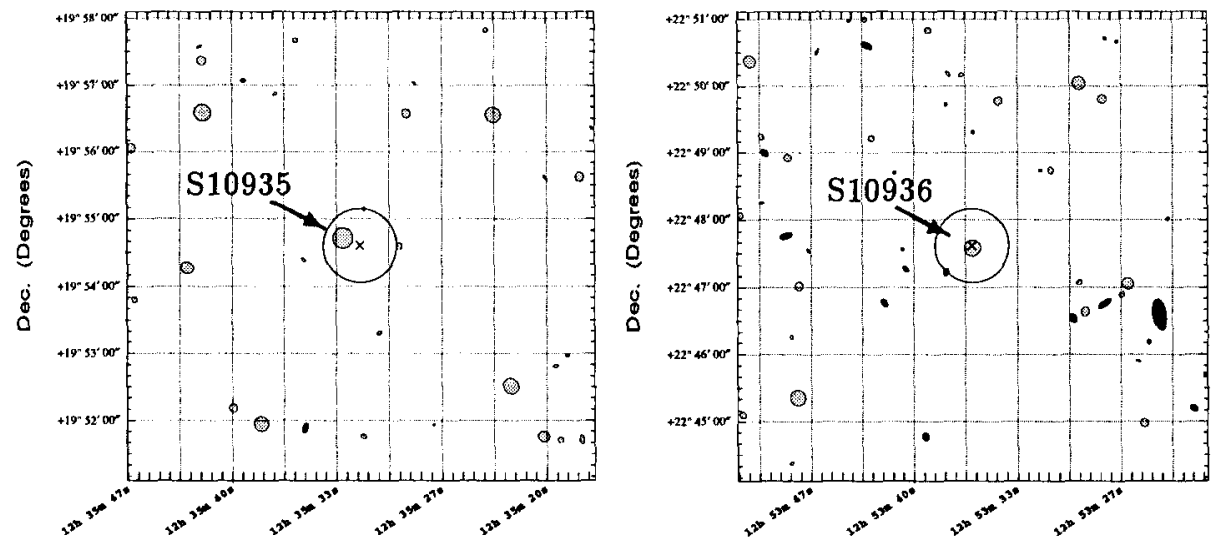

R.A. (Hours)
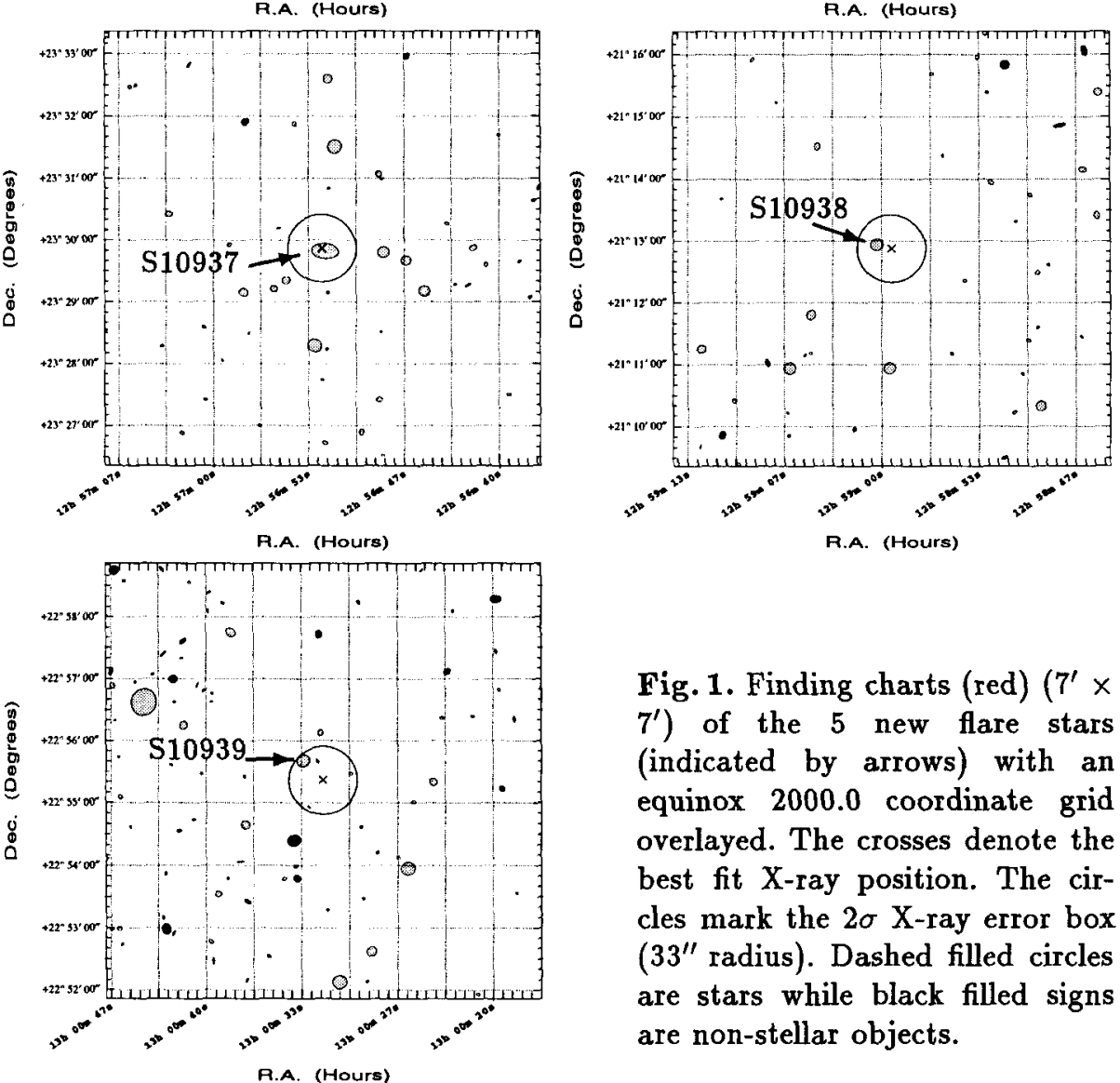

R.A. (Hours)

Fig. 1. Finding charts (red) $\left(7^{\prime} \times\right.$ $7^{\prime}$ ) of the 5 new flare stars (indicated by arrows) with an equinox 2000.0 coordinate grid overlayed. The crosses denote the best fit X-ray position. The circles mark the $2 \sigma \mathrm{X}$-ray error box (33" radius). Dashed filled circles are stars while black filled signs are non-stellar objects. 
The flare stars of Table 2 have been found according to the classical method for searching for eruptions (Haro 1968) by taking multiple exposures. In principle, this method allows to calculate the discovery probability of flare stars according to Poisson statistics (Ambartsumian 1970). In practice, the determination of this very low probability is rather laborious and gives reasonable results only with large samples.

Though statistics is small, the above reported discovery of 5 new flare stars approximately doubles the number of known flare stars in this high galactic latitude field. The fact that none of the X-ray selected flare stars was optically discovered prior to $R O S A T$, is a further indication of a very small discovery probability of faint flare stars. This implies a high frequency and, because of the low luminosity, a large space density. However, quantitative conclusions cannot be drawn without investigations of additional fields (at different galactic latitudes).

It is clear, however, that the use of a complete $\mathrm{X}$-ray selected sample of sources represents an effective way of identifying optically faint, active dwarf stars, (and in particular if a large number of archival plates can be used). Further identification work of the ROSAT All-Sky-Survey sources promises many more flare stars to be discovered.

Acknowledgement: This work has been supported by funds of the German Bundesministerium für Forschung und Technologie under grant 052 SO524 (GAR) and FKZ 50 OR 9201 (JG). We are grateful to R. McMahon for installing the software used for the APM finding charts. The ROSAT project is supported by the German Bundesministerium für Forschung und Technologie (BMFT/DARA) and the Max-Planck-Society.

\section{References}

Ambartsumian V., 1970, Astrofizika 6, 31

Argue A.N., Kenworthy C.M., 1968, Observatory 88, 109

Haro G., 1968, Stars and Stellar Systems 7, 141 